\title{
Complex analysis of the p53 tumor suppressor in lung carcinoma
}

\author{
JANA SMARDOVA $^{1,2}$, KVETOSLAVA LISKOVA $^{1}$, BARBORA RAVCUKOVA ${ }^{3}$, JITKA MALCIKOVA ${ }^{4}$, \\ JITKA HAUSNEROVA ${ }^{1}$, MILUSE SVITAKOVA ${ }^{1}$, RENATA HRABALKOVA ${ }^{1}$, LENKA ZLAMALIKOVA ${ }^{1}$, \\ KATERINA STANO-KOZUBIK ${ }^{4}$, IVONA BLAHAKOVA ${ }^{4}$, JANA SPELDOVA ${ }^{5}$, \\ JIRI JARKOVSKY ${ }^{6}$ and JAN SMARDA ${ }^{2}$

\begin{abstract}
${ }^{1}$ Department of Pathology, University Hospital; ${ }^{2}$ Department of Experimental Biology, Faculty of Science, Masaryk University; ${ }^{3}$ Molecular Genetics Laboratory, Centre for Cardiovascular Surgery and Transplantation;

${ }^{4}$ Central European Institute of Technology (CEITEC), Masaryk University; ${ }^{5}$ Department of Respiratory Diseases and TB, University Hospital; ${ }^{6}$ Institute of Biostatistics and Analyses, Faculty of Medicine, Masaryk University, Brno, Czech Republic
\end{abstract}

Received October 7, 2015; Accepted November 10, 2015

DOI: 10.3892/or.2015.4533

\begin{abstract}
Lung cancer is the leading cause of cancer-related deaths worldwide. The p53 tumor suppressor is a transcription factor controlling expression of its target genes in response to various stress stimuli. Mutations of the TP53 gene occur very frequently in lung carcinomas and they play an important role in both oncogenic transformation of lung epithelial cells and lung carcinoma progression. We determined the TP53 status in 42 samples of squamous cell lung carcinoma (SQCC) and 56 samples of lung adenocarcinoma (AC) by the functional analysis FASAY and its variant called split assay. Altogether, we detected 64 TP53 mutations in 63 patients and analyzed them by cDNA and gDNA sequencing. The TP53 mutations were found in $76.2 \%(32 / 42)$ of SQCC cases, and $55.4 \%(31 / 56)$ of ACs. Immunoblotting revealed the p53 protein accumulation in 18 samples (42.9\%) among SQCC cases and 19 samples (33.9\%) among AC cases. Using fluorescence in situ hybridization we detected loss of the TP53-specific 17p13.3 locus in 23 from 41 analyzed SQCC samples (56.1\%) and in 20 from 54 analyzed AC samples (37.0\%). We did not find any statistically significant differences in overall and disease-free survival in relation to TP53 status.
\end{abstract}

\section{Introduction}

Lung cancer is the leading cause of cancer-related deaths worldwide $(1,2)$. Despite improvements in therapy, the 5-year survival rate for all patients diagnosed with lung cancer remains as low as $15-20 \%$. Approximately $75-80 \%$ of human lung

Correspondence to: Professor Jana Smardova, Department of Pathology, University Hospital, Jihlavska 20, 62500 Brno, Czech Republic

E-mail: janasmarda@seznam.cz

Key words: lung adenocarcinoma, lung squamous cell carcinoma, p53 tumor suppressor, FASAY, FISH, TP53 mutation cancers are non-small cell lung cancers (NSCLC). NSCLC represents a heterogeneous group of cancers and includes two major forms: lung squamous cell carcinoma (SQCC) and lung adenocarcinoma (AC).

Development of lung cancers involves formation of multiple genetic aberrations, including those affecting the p53 tumor suppressor. p53 mediates an adequate reaction of cells to stress. It functions as a transcription factor responding to various stress signals by controlling expression of its target genes. The p53 protein then participates in control of cell cycle, DNA repair, apoptosis, senescence and maintaining of genome integrity $(3,4)$. Mutations and allelic deletions of the TP53 gene are the most frequent genetic alterations detected in human tumors, including lung cancers. Inactivation of p53 usually results from missense mutations in the core region of the TP53 gene coding for the sequence-specific DNA-binding domain. Due to inability to transactivate its negative regulator, the E3-ubiquitin ligase MDM2 (5-7), the mutant p53 protein often accumulates in tumor cells.

Mutations of the TP53 gene are very frequent in lung carcinomas and they play an important role in both oncogenic transformation of lung epithelial cells and lung carcinoma progression (8). There are multiple lines of evidence documenting that the presence of TP53 aberrations worsen the prognosis of patients with lung cancer (8-11). Frequency of the TP53 aberrations in lung cancers has been tested repeatedly and showed higher frequency of the TP53 mutations in lung SQCC (50-70\%) than in lung AC ( 40\%) (11-13). Integrated and comprehensive genomic characterization was performed both in AC and squamous cell lung cancers $(14,15)$. By detailed analysis of 188 cases of lung AC, 26 genes were identified as mutated at significantly high frequencies and thus they are probably involved in development of the disease. Among them, TP53 was mutated in 66 cases (34\%). Notably, the rate of missense mutations was rather low $(65.2 \%)$ and some specific mutations were detected repeatedly, namely p.G266* (4x), p.R273L (3x), p.R282W (3x), and others (14). For squamous cell lung cancers, 178 cases were analyzed and statistically recurrent mutations in 11 genes were found. One of them, the TP53 gene was mutated in $90 \%$ of the samples (15). 
In the present study, we analyzed the TP53 status in 42 samples of lung SQCC and 56 samples of lung AC by the functional analysis FASAY and its variant called split assay followed by cDNA and gDNA sequencing. Each sample was also tested for the p53 protein accumulation by immunoblotting and loss of the TP53-specific 17p13.3 locus by fluorescence in situ hybridization. We examined relationship between the TP53 status and the disease outcome.

\section{Materials and methods}

Clinical materials. Ninety-eight patients (37 females and 61 males) were included in the study. They underwent surgical removal of the tumor tissue in University Hospital in Brno from March 2006 to January 2014. All samples were classified according to WHO guidelines. Forty-two patients (9 females and 33 males) were diagnosed with spinocellular lung carcinoma (cases labeled S1 to S42; Table I). The average age of patients at the time of surgical intervention was 65.5 years, ranging from 40 to 79 years. Fifty-six patients ( 28 females and 28 males) were diagnosed with AC (cases labeled A1 to A56; Table II). The average age of patients at the time of surgical intervention was 66.5 years, ranging from 41 to 86 years. Among SQCC patients 9 (24.3\%) were non-smokers, 9 (24.3\%) ex-smokers and 19 (51.4\%) smokers. Among AC patients the ratio was $42.0 \%$ (21 non-smokers), $20.0 \%$ (10 ex-smokers) and $38.0 \%$ (19 smokers). For all included patients, fresh frozen tissue samples as well as formalin-fixed, paraffin-embedded tumor tissue blocks (FFPE) were available. Patients were included in the present study only after signing the informed consent form approved by the Ethics Committee of the hospital.

FASAY and split assay. FASAY was performed as previously described $(16,17)$ with small modifications. Total RNA was extracted using NucleoSpin RNA kit (New England BioLabs Inc., Ipswich, MA, USA) and stored at $-80^{\circ} \mathrm{C}$ until further processing. cDNA was synthesized by ProtoScript ${ }^{\circledR}$ II (New England BioLabs Inc.) using primer oligo $(\mathrm{dT})_{12}$. PCR was performed using primers $\mathrm{P} 3$ (5'-CCT-TGC-CGT-CCC-AAG-CAA-TGG-ATG-AT-3'); P4 (5'-ACC-CTT-TTT-GGA-CTT-CAG-GTG-GCT-GGA-GT-3'), and Phusion DNA Polymerase (New England BioLabs Inc.). Yeast cells were co-transformed with the PCR product, linearized pSS16 plasmid and salmon sperm DNA carrier (Life Technologies Inc., Carlsbad, CA, USA) by the lithium acetate procedure (18). Transformed yeast cells were plated on minimal medium lacking leucine and with $5 \mu \mathrm{g} / \mathrm{ml}$ of adenine, followed by incubation at $35^{\circ} \mathrm{C}$ for $2-3$ days, and then at room temperature for 2-3 days. For split assay, PCR of the TP53 5'-part was performed with primers P3 and P17 (5'-GCC-GCC-CAT-GCA-GGA-ACT-GTT-ACA-CAT-3'); the 3'-part with primers P4 and P16 (5'-GCG-ATG-GTCTGG-CCC-CTC-CTC-AGC-ATC-TTA-3'). Yeast cells were transformed with linearized vectors pFW35 and pFW34 (19).

FASAY deduces the functional status of p53 from the color of colonies of transformed yeast cells. Expression of functional p53 results in formation of large white colonies, inactive p53 leads to smaller red ones. The background frequency of red yeast colonies typically does not exceed $10 \%$. Thus, samples providing $<10 \%$ of red colonies are considered to contain only wild-type TP53 alleles, while samples providing $>10 \%$ of red colonies are suspicious to possess a clonal TP53 mutation. The ratio of red colonies scoring between 10 and $20 \%$ can result from a presence of clonal TP53 mutation in rather small fraction of cells or from increased rate of RNA degradation. To distinguish these two possibilities, version of FASAY, called split assay was established. In the split assay, the 5'- and 3'-parts of the TP53 cDNA were tested separately (19).

Purification of the plasmids from transformed yeast cells and sequencing of the TP53 cDNA. Yeast cells from individual yeast colonies were harvested, resuspended in TSN (2\% Triton X-100, 1\% SDS, $100 \mathrm{mM} \mathrm{NaCl}, 10 \mathrm{mM}$ Tris $\mathrm{pH} 8.0$, $1 \mathrm{mM}$ EDTA), and grinded by vortexing with glass beads; plasmid DNA was extracted by phenol/chloroform procedure. The TP53 cDNA was amplified using the P3 and P4 primers and Taq polymerase (Life Technologies Inc.) and subjected to agarose gel electrophoresis. The PCR product was purified by MinElute PCR purification kit (Qiagen, Hilden, Germany) and sequenced by BigDye terminator v3.1 cycle sequencing kit using ABI PRISM 3130 Genetic Analyzer (both from Applied Biosystems, Darmstadt, Germany).

Isolation and sequencing of the TP53 gDNA. Genomic DNA was isolated from FFPE blocks or from the frozen tumor samples using Purogene DNA Isolation kit (Gentra Systems, Minneapolis, MN, USA) according to the manufacturer's instructions. The TP53 exons were amplified by PCR and automated fluorescent sequencing was performed using BigDye terminator kit and ABI 3100 sequencer (Applied Biosystems, Carlsbad, CA, USA). Either a picked exon of interest or all exons 2-11 were analyzed. Primers and conditions were adopted from IARC TP53 database (20).

Immunoblotting. Tissue samples were lysed in $150 \mathrm{mM} \mathrm{NaCl}$, $50 \mathrm{mM}$ NaF, $50 \mathrm{mM}$ Tris (pH 8.0), $5 \mathrm{mM}$ EDTA, $1 \%$ NP40 and $1 \mathrm{mM}$ phenylmethylsulfonyl fluoride in ice for $30 \mathrm{~min}$, and the cell extract was centrifuged at $17,000 \mathrm{x}$ g for $30 \mathrm{~min}$ to remove cell debris. Protein concentration was measured by the Bradford assay. Solubilized proteins were resolved by $10 \%$ SDS-PAGE and transferred onto a nitrocellulose membrane. Blots were blocked in $0.1 \%$ Tween-20 and 5\% low-fat milk in PBS for $1 \mathrm{~h}$ and probed with anti-p53 mouse monoclonal antibody DO-1 (Dako, Glostrup, Denmark) at $4^{\circ} \mathrm{C}$. Blots were developed with Dako peroxidase-conjugated rabbit anti-mouse immunoglobulin (Dako) using the ECL chemiluminescence detection kit (GE Healthcare UK Ltd., Little Chalfont, UK).

Fluorescence in situ hybridization (FISH). FISH was performed in tissue sections prepared from FFPE blocks. For the TP53-specific locus analysis, the ZytoLight ${ }^{\circledR}$ SPEC TP53/CEN17 Dual Color Probe was used (ZytoVision GmbH, Bremerhaven, Germany). Hybridization was performed according to the manufacturer's instructions. Images were scanned by DM 5500 B microscope equipped with Leica DFC 290 HD camera. Fluorescence signals were analyzed using Leica LAS AF software (Leica Microsystems GmbH, Wetzlar, Germany). Cells (50-100) per case were analyzed. The cut-off level was defined by the mean value plus three 
Table I. Summary of the TP53 status analyses in samples of squamous cell lung carcinoma.

\begin{tabular}{|c|c|c|c|c|c|c|c|}
\hline \multirow[b]{2}{*}{ Case } & \multirow[b]{2}{*}{ Gender/age } & \multirow[b]{2}{*}{ FASAY $^{\mathrm{a}}$} & \multicolumn{2}{|c|}{ DNA sequencing } & \multirow[b]{2}{*}{ TP53 status } & \multirow[b]{2}{*}{$\mathrm{FISH}^{\mathrm{b}}$} & \multirow[b]{2}{*}{ IB } \\
\hline & & & cDNA & gDNA & & & \\
\hline 1 & $\mathrm{M} / 59$ & 70.3 & $\mathrm{r} .1024 \mathrm{c}>\mathrm{u}^{\mathrm{c}, \mathrm{d}}$ & c. $1024 C>T^{f}$ & c. $1024 \mathrm{C}>\mathrm{T}$ p.R342* & 27 & - \\
\hline 4 & $\mathrm{M} / 79$ & WT & ND & $\mathrm{WT}^{\mathrm{f}}$ & WT & 78 & - \\
\hline 5 & $\mathrm{M} / 76$ & 95.6 & $\mathrm{r} .464 \mathrm{c}>\mathrm{a}^{\mathrm{c}}$ & ND & c. $464 \mathrm{C}>$ A p. $\mathrm{T} 155 \mathrm{~N}$ & 46 & ++ \\
\hline 6 & $\mathrm{~F} / 65$ & 20.1 & r.921-939del ${ }^{\mathrm{d}}$ & c. $920-1 \mathrm{G}>\mathrm{A}^{\mathrm{e}}$ & c. $.920-1 \mathrm{G}>$ A p.L308Pfs $* 31$ & 34 & - \\
\hline 7 & $\mathrm{M} / 77$ & 32.8 & r.207delu ${ }^{\mathrm{d}}$ & c.207delT ${ }^{f}$ & c.207delT p.A70Lfs $* 53$ & 27 & - \\
\hline 8 & $\mathrm{M} / 67$ & 82.4 & $\mathrm{r} .461 \mathrm{~g}>\mathrm{u}^{\mathrm{c}}$ & ND & c. $461 \mathrm{G}>\mathrm{T}$ p.G154C & ND & +++ \\
\hline 9 & $\mathrm{~F} / 68$ & WT & ND & ND & WT & 7 & - \\
\hline 10 & $\mathrm{~F} / 55$ & WT & ND & $\mathrm{WT}^{\mathrm{f}}$ & WT & 33 & - \\
\hline 11 & $\mathrm{M} / 53$ & 28.1 & r. $488 \mathrm{a}>\mathrm{g}^{\mathrm{c}}$ & ND & c. $488 \mathrm{~A}>\mathrm{G}$ p.. $\mathrm{Y} 163 \mathrm{C}$ & 9 & - \\
\hline 12 & $\mathrm{M} / 50$ & 71.2 & $\mathrm{r} .764 \mathrm{u}>\mathrm{g}^{\mathrm{c}}$ & c. $764 \mathrm{~T}>\mathrm{G}^{\mathrm{f}}$ & c.764T>G p.I255S & 7 & +++ \\
\hline 13 & $\mathrm{M} / 63$ & 34.9 & r.422dupg ${ }^{\mathrm{d}}$ & c. $422 \mathrm{dupG}^{\mathrm{e}}$ & c.422dupG p.C141Wfs*7 & 6 & - \\
\hline 14 & $\mathrm{M} / 69$ & WT & ND & ND & WT & 37 & - \\
\hline 15 & $\mathrm{M} / 69$ & 74.7 & $\begin{array}{l}\text { r. }[782+2 \mathrm{u}>\mathrm{g} ; \\
782 \_783 i n \mathrm{in} 78 \\
\left.2+1 \_783-1\right]^{\mathrm{c}, \mathrm{d}}\end{array}$ & c. $782+2 \mathrm{~T}>\mathrm{G}^{\mathrm{f}}$ & $\begin{array}{l}\text { c. } 782+2 T>G \\
\text { p.S261Rfs*63 }\end{array}$ & 13 & - \\
\hline 17 & $\mathrm{M} / 48$ & 18.4 & $\mathrm{r} .734 \mathrm{~g}>\mathrm{u}^{\mathrm{d}}$ & ND & c.734G>T p.G245V & 68 & - \\
\hline 18 & $\mathrm{M} / 62$ & 85.2 & r.313_375del ${ }^{\mathrm{c}, \mathrm{d}}$ & c. $314 \mathrm{G}>\mathrm{T}^{\mathrm{f}}$ & c.314G>T p.G105_T125del & 6 & ++ \\
\hline 19 & $\mathrm{M} / 68$ & 41.7 & $\mathrm{r} .764 \mathrm{u}>\mathrm{a}^{\mathrm{c}}$ & c. $764 \mathrm{~T}>\mathrm{A}^{\mathrm{f}}$ & c.764T $>$ A p.I255N & 5 & +++ \\
\hline 20 & $\mathrm{M} / 64$ & 29.6 & r. $746 \mathrm{~g}>\mathrm{c}^{\mathrm{c}}$ & ND & c.746G >C p.R249T & 76 & +++ \\
\hline 21 & $\mathrm{~F} / 40$ & WT & ND & ND & WT & 2 & - \\
\hline 22 & $\mathrm{~F} / 63$ & WT & ND & $\mathrm{WT}^{\mathrm{f}}$ & WT & 11 & + \\
\hline 23 & $\mathrm{M} / 61$ & 73.5 & $\mathrm{r} .848 \mathrm{~g}>\mathrm{c}^{\mathrm{c}}$ & c. $.848 \mathrm{G}>\mathrm{C}^{\mathrm{f}}$ & c. $848 \mathrm{G}>\mathrm{C}$ p.R283P & 11 & - \\
\hline 24 & $\mathrm{M} / 57$ & 99.2 & $\mathrm{r} .706 \mathrm{u}>\mathrm{g}^{\mathrm{c}}$ & c. $706 \mathrm{~T}>\mathrm{G}^{\mathrm{e}}$ & c.706T>G p.Y236D & 9 & +++ \\
\hline 25 & $\mathrm{M} / 68$ & WT & ND & ND & WT & 81 & - \\
\hline 26 & $\mathrm{M} / 67$ & 15.4 & r.403delu ${ }^{\mathrm{d}}$ & c. $403 \mathrm{del} \mathrm{T}^{\mathrm{f}}$ & c.403delT p.C137Afs $* 35$ & 8 & - \\
\hline 27 & $\mathrm{M} / 66$ & 95.9 & $\mathrm{r} .380 \mathrm{c}>\mathrm{u}^{\mathrm{c}}$ & ND & c. $380 \mathrm{C}>\mathrm{T}$ p.S127F & 12 & +++ \\
\hline 87 & $\mathrm{M} / 65$ & 86.2 & $\mathrm{r} .848 \mathrm{~g}>\mathrm{c}^{\mathrm{c}}$ & ND & c. $848 \mathrm{G}>\mathrm{C}$ p.R283P & 4 & +++ \\
\hline 88 & $\mathrm{M} / 60$ & 94.1 & $\mathrm{r} .404 \mathrm{~g}>\mathrm{u}^{\mathrm{c}}$ & c. $404 \mathrm{G}>\mathrm{T}^{\mathrm{e}}$ & c. $404 \mathrm{G}>\mathrm{T}$ p.C135F & 41 & +++ \\
\hline 89 & $\mathrm{~F} / 65$ & 92.6 & r. $.359 a>u^{c}$ & c. $359 \mathrm{~A}>\mathrm{T}^{\mathrm{e}}$ & c. $359 \mathrm{~A}>\mathrm{T}$ p.K120M & 36 & +++ \\
\hline 90 & $\mathrm{M} / 60$ & 88.0 & $\mathrm{r} .422 \mathrm{~g}>\mathrm{a}^{\mathrm{c}}$ & c. $422 \mathrm{G}>\mathrm{A}^{\mathrm{e}}$ & c. $422 \mathrm{G}>\mathrm{A}$ p.C141Y & 29 & ++ \\
\hline 91 & $\mathrm{~F} / 58$ & 93.9 & $\mathrm{r} .820 \mathrm{~g}>\mathrm{u}^{\mathrm{c}}$ & c. $820 \mathrm{G}>\mathrm{T}^{\mathrm{e}}$ & c. $820 \mathrm{G}>\mathrm{T}$ p.V274F & 46 & +++ \\
\hline 92 & $\mathrm{M} / 63$ & WT & $\mathrm{ND}$ & ND & WT & 28 & - \\
\hline 93 & $\mathrm{M} / 66$ & 92.7 & $\mathrm{r} .752 \mathrm{u}>\mathrm{a}^{\mathrm{c}}$ & c. $752 \mathrm{~T}>\mathrm{A}^{\mathrm{f}}$ & c. $752 \mathrm{~T}>\mathrm{A}$ p.I251N & 14 & +++ \\
\hline 94 & $\mathrm{M} / 66$ & 21.3 & r.637c $>u^{d}$ & c. $637 \mathrm{C}>\mathrm{T}^{\mathrm{f}}$ & c. $637 \mathrm{C}>\mathrm{T}$ p.R213* & 60 & - \\
\hline 95 & $\mathrm{M} / 64$ & WT & ND & ND & WT & 41 & - \\
\hline 96 & $\mathrm{M} / 71$ & 26.8 & $\mathrm{r} .461 \mathrm{~g}>\mathrm{u}^{\mathrm{d}}$ & c. $461 \mathrm{G}>\mathrm{T}^{\mathrm{f}}$ & c. $461 \mathrm{G}>\mathrm{T}$ p.G154V & 68 & - \\
\hline 97 & $\mathrm{M} / 69$ & 24.7 & $\begin{array}{l}\text { r.176_375del } \\
(3 / 15)^{\mathrm{c}, \mathrm{g}} \\
\text { r.[375+5g }>\mathrm{a} ; \\
375 \_376 \text { ins } 37 \\
\left.5+1 \_375+109\right] \\
(3 / 15)^{\mathrm{c}, \mathrm{g}} \\
\text { r.[375+5g } 7 \text { a; } \\
375 \_376 \text { ins } 37 \\
\left.5+1 \_376-1\right] \\
(3 / 15)^{\mathrm{c}, \mathrm{g}}\end{array}$ & c. $375+5 \mathrm{G}>\mathrm{A}^{\mathrm{f}}$ & $\begin{array}{l}\text { c. } 375+5 G>A \\
\text { p.G59Vfs*23 } \\
(3 / 15)^{d} \\
\text { p.Y126Vfs*59 } \\
(3 / 15)^{\text {d }} \\
\text { p.Y126Vfs*49 } \\
(3 / 15)^{d}\end{array}$ & 10 & - \\
\hline 98 & $\mathrm{M} / 75$ & 98.6 & $\mathrm{r} .832 \mathrm{c}>\mathrm{u}^{\mathrm{c}}$ & c. $832 \mathrm{C}>\mathrm{T}^{\mathrm{f}}$ & c. $832 \mathrm{C}>\mathrm{T}$ p.P278S & 6 & +++ \\
\hline 99 & $\mathrm{~F} / 43$ & WT & $\mathrm{ND}$ & ND & WT & 30 & - \\
\hline 100 & $\mathrm{M} / 68$ & 20.4 & $\begin{array}{l}\text { r.418_422deli } \\
\text { nsgcccuu }^{\text {d }}\end{array}$ & $\begin{array}{l}\text { c.418_422delins6G } \\
\text { CCCTT }^{\text {f }}\end{array}$ & $\begin{array}{l}\text { c. } 418 \_422 \text { delins } \\
\text { GCCCTT p.T140Afs*9 }\end{array}$ & 26 & - \\
\hline 101 & $\mathrm{~F} / 76$ & 81.0 & $\mathrm{r} .473 \mathrm{~g}>\mathrm{u}^{\mathrm{c}}$ & c. $473 \mathrm{G}>\mathrm{T}^{\mathrm{f}}$ & c. $473 \mathrm{G}>\mathrm{T}$ p.R158L & 79 & +++ \\
\hline
\end{tabular}


Table I. Continued.

\begin{tabular}{|c|c|c|c|c|c|c|c|}
\hline \multirow[b]{2}{*}{ Case S } & \multirow[b]{2}{*}{ Gender/age } & \multirow[b]{2}{*}{ FASAY $^{\mathrm{a}}$} & \multicolumn{2}{|c|}{ DNA sequencing } & \multirow[b]{2}{*}{ TP53 status } & \multirow[b]{2}{*}{ FISH $^{\mathrm{b}}$} & \multirow[b]{2}{*}{ IB } \\
\hline & & & cDNA & gDNA & & & \\
\hline 102 & $\mathrm{M} / 70$ & 93.1 & $\mathrm{r} .461 \mathrm{~g}>\mathrm{u}^{\mathrm{c}}$ & c. $461 \mathrm{G}>\mathrm{T}^{\mathrm{f}}$ & c. $461 \mathrm{G}>\mathrm{T}$ p.G154V & 42 & +++ \\
\hline 103 & $\mathrm{M} / 76$ & 11.0 & $\mathrm{WT}^{\mathrm{d}}$ & c. $.511 \mathrm{G}>\mathrm{T}^{\mathrm{f}}$ & c. $511 \mathrm{G}>\mathrm{T}$ p.E171* & 44 & - \\
\hline 104 & $\mathrm{M} / 77$ & 96.3 & $\begin{array}{l}\text { r.739_752deli } \\
\text { nsgg }^{\text {c,d }}\end{array}$ & c.739_752delinsGG ${ }^{\mathrm{f}}$ & $\begin{array}{l}\text { c.739_752delinsGG } \\
\text { p.N247_I251delinsG }\end{array}$ & 10 & +++ \\
\hline
\end{tabular}

M, male; F, female; IB, immunoblotting; ND, not done; WT, wild-type; ${ }^{\mathrm{a}} \%$ of red colonies; ${ }^{\mathrm{b}} \%$ of positive cells; ${ }^{\mathrm{c}} \mathrm{cDNA}$ prepared from tumor tissue; ${ }^{\mathrm{d}} \mathrm{CDNA}$ isolated from yeast colonies; ${ }^{\mathrm{e}} \mathrm{gDNA}$ isolated from FFPE; ${ }_{\mathrm{f} D N A}$ isolated from frozen tumor tissue; ${ }^{\mathrm{g}}$ fraction of positive colonies.

times the SD of the frequency of control cells exhibiting one red and two green signals $(9.6 \%)$.

Statistical analyses. Standard descriptive statistics was used; median supplemented with min-max range for continuous variables and absolute and relative frequencies for categorical variables. The influence of patient characteristics on their survival was analyzed using the Cox proportional hazards model and described using hazard ratios, their $95 \%$ confidence interval and statistical significance. Analysis was computed using SPSS 22.0.0.1 (IBM Corporation, 2014).

\section{Results}

Assessment of the p53 status by FASAY and split assay. We used FASAY to determine the TP53 status of all 98 enrolled samples and assessed the ratio of red colonies. Among 42 samples of SQCCs, 7 samples scored under the $10 \%$ background level and were classified as bearing the standard TP53 variant. Split assay was performed in all samples exceeding the cut-off $10 \%$ level. Three samples only slightly exceeding the background level in FASAY (10.8-11.4\%) scored under the cut-off $10 \%$ level in both parts of split assay. Therefore, these samples were also classified as having the standard TP53 variant. Remaining 32 samples were suspected to contain a clonal TP53 mutation and they underwent further analyses (Table I). The same procedure was performed for 56 enrolled lung AC samples. Twenty-five samples scored under the background level, while the remaining 31 samples were assigned for further analyses (Table II).

Sequencing of the TP53 cDNA. All 63 cases positive for TP53 mutation as determined by FASAY/split assay were analyzed by cDNA sequencing. Two strategies were used. In 45 cases, usually with higher ratio of red colonies in FASAY, cDNA to be sequenced was prepared directly from tumor tissues. In 29 cases, the TP53 expression vector was recovered from 3 to 10 red yeast colonies per case, and this cDNA was used as template for DNA sequencing. In 11 cases, both approaches were combined with affirmative results (Tables I and II). With exception of three cases, unambiguous clonal TP53 mutations were identified in each sample (60/63): 29 cases of $\mathrm{AC}$ and 31 cases of SQCC. No clonal mutation was found in the AC case A53 with score $16.8 \%$ of red colonies in FASAY. In AC case A35 the results of FASAY and split assay were only slightly above the cut-off level (score $14.7 \%$ in FASAY and 6.1/13.4 in split assay) and no potential clonal mutation was suggested by the TP53 cDNA sequencing of randomly picked eight yeast colonies. Each colony contained different TP53 mutation, one of them was nonsense p.K321* mutation. We repeated the whole procedure and analyzed 10 more yeast colonies. The mutation p.K321* was found in two other colonies and was subsequently confirmed by gDNA analyses. In the SQCC case $\mathrm{S} 41$ (score $11.0 \%$ in FASAY) the presence of missense mutation p.G154V was proposed as it was detected in 2 from 8 red colonies tested. Although the mutation was not confirmed by subsequent gDNA analysis, the nonsense mutation p.E171* was clearly disclosed instead.

Sequencing of the TP53 gDNA. Two main strategies were used for TP53 gDNA sequencing. First, the gDNA was isolated from FFPE blocks, the TP53 exons 2-11 were amplified by PCR and sequenced. Fifteen cases with mutated TP53 and five randomly chosen cases with standard TP53 as determined by cDNA analyses were subjected to this procedure. In all 15 positive cases, the same mutation identified by cDNA sequencing was confirmed. Four cases lacking TP53 mutation scored standard also by gDNA sequencing. In AC sample A23 which was classified as standard by FASAY (scoring 9.2\%), the presence of two independent TP53 mutations was revealed (A and B). One mutation A23-A: p.G154V was confirmed by sequencing of cDNA isolated from background red yeast colonies obtained by FASAY (in 3/9 colonies tested), the other mutation (A23-B: c.557_558ins17N) was not traced in this way (0/9).

Second, the gDNA was isolated from frozen tumor tissues and then subjected to the sequencing analysis. For 34 TP53 mutation positive samples revealed by cDNA sequencing (including the cases A35 and S41) or gDNA sequencing from FFPE samples (case A23 A and B) only the relevant TP53 exons were PCR amplified and sequenced. In all cases, the proposed mutations were confirmed. The same technical procedure comprising exons 2-11 was employed in seven other cases with the wild-type TP53 output determined by FASAY. They included six cases carrying deletion of the TP53 locus assessed by FISH analysis (see below). No TP53 mutation was disclosed in any of these samples. 
Table II. Summary of the TP53 status analyses in samples of lung adenocarcinoma.

\begin{tabular}{|c|c|c|c|c|c|c|c|}
\hline \multirow[b]{2}{*}{ Case A } & \multirow[b]{2}{*}{ Gender/age } & \multirow[b]{2}{*}{ FASAY $^{\mathrm{a}}$} & \multicolumn{2}{|c|}{ DNA sequencing } & \multirow[b]{2}{*}{ TP53 status } & \multirow[b]{2}{*}{$\mathrm{FISH}^{\mathrm{b}}$} & \multirow[b]{2}{*}{ IB } \\
\hline & & & cDNA & gDNA & & & \\
\hline 28 & $\mathrm{M} / 62$ & WT & ND & ND & WT & - & \\
\hline 30 & $\mathrm{~F} / 78$ & WT & ND & ND & WT & - & \\
\hline 31 & $\mathrm{~F} / 71$ & WT & ND & ND & WT & - & \\
\hline 32 & $\mathrm{M} / 73$ & WT & ND & $\mathrm{WT}^{\mathrm{f}}$ & WT & - & \\
\hline 33 & $\mathrm{M} / 65$ & WT & ND & $\mathrm{WT}^{\mathrm{f}}$ & WT & - & \\
\hline 34 & $\mathrm{M} / 57$ & 92.3 & $\mathrm{r} .596 \mathrm{~g}>\mathrm{u}^{\mathrm{c}}$ & c. $596 \mathrm{G}>\mathrm{T}^{\mathrm{f}}$ & c. $596 \mathrm{G}>\mathrm{T}$ p.G199V & 10 & +++ \\
\hline 35 & $\mathrm{M} / 67$ & 97.8 & $\begin{array}{l}\text { r.313_375del } \\
(11 / 13)^{\mathrm{c}, \mathrm{d}, \mathrm{g}} \\
\text { r.314g>u } \\
(1 / 13)^{\mathrm{c}, \mathrm{d}, \mathrm{g}}\end{array}$ & c. $314 \mathrm{G}>\mathrm{T}^{\mathrm{f}}$ & $\begin{array}{l}\text { c. } 314 G>T \quad 7 \\
\text { p.G105_T125del } \\
(11 / 13)^{\mathrm{d}} \\
\text { p.G105V }(1 / 13)^{\mathrm{d}}\end{array}$ & - & \\
\hline 36 & $F / 76$ & WT & ND & $\mathrm{WT}^{\mathrm{f}}$ & WT $\quad 41$ & - & \\
\hline 37 & $\mathrm{~F} / 68$ & 58.3 & r. $358 \mathrm{a}>\mathrm{u}^{\mathrm{c}, \mathrm{d}}$ & c. $358 \mathrm{~A}>\mathrm{T}^{\mathrm{f}}$ & c. $.358 \mathrm{~A}>\mathrm{T}$ p.K120* & 8 & - \\
\hline 38 & $\mathrm{M} / 54$ & 18.9 & r.461 delg & c. $461 \mathrm{delG}^{\mathrm{f}}$ & c.461delG p.G154Afs*15 & 18 & - \\
\hline 39 & $\mathrm{~F} / 67$ & WT & ND & ND & $\begin{array}{ll}\text { WT } & 7\end{array}$ & - & \\
\hline 40 & $\mathrm{~F} / 68$ & 83.4 & $\mathrm{r} .743 \mathrm{~g}>\mathrm{a}^{\mathrm{c}}$ & c. $743 \mathrm{G}>\mathrm{A}^{\mathrm{f}}$ & c.743G>A p.R248Q & 14 & +++ \\
\hline 41 & $\mathrm{M} / 68$ & 82.9 & $\mathrm{r} .820 \mathrm{~g}>\mathrm{u}^{\mathrm{c}}$ & $\mathrm{c} .820 \mathrm{G}>\mathrm{T}^{\mathrm{f}}$ & c.820G $>$ T p.V274F & 6 & +++ \\
\hline 42 & $\mathrm{~F} / 57$ & 89.2 & r.733g $>\mathrm{a}^{\mathrm{c}}$ & c. $733 \mathrm{G}>\mathrm{A}^{\mathrm{f}}$ & c. $733 \mathrm{G}>$ A p.G245S & 25 & +++ \\
\hline 43 & $\mathrm{M} / 61$ & 88.3 & r.743_744gg $>\mathrm{uu}^{\mathrm{d}}$ & c.743_744GG>TT ${ }^{\mathrm{f}}$ & $\begin{array}{l}\text { c.743_744GG>TT } \\
\text { p.R248L }\end{array}$ & 13 & +++ \\
\hline 44 & $\mathrm{M} / 60$ & 27.9 & r.1013delu ${ }^{\mathrm{d}}$ & c. $1013 \mathrm{delT}^{\mathrm{f}}$ & c.1013delT p.F338Sfs*7 & 20 & - \\
\hline 45 & $\mathrm{~F} / 63$ & 19.4 & $\mathrm{r} .839 \mathrm{~g}>\mathrm{c}^{\mathrm{d}}$ & ND & c.839G >C p.R280T & 18 & +++ \\
\hline 46 & $\mathrm{~F} / 67$ & 27.3 & $\mathrm{r} .406 \mathrm{c}>\mathrm{g}^{\mathrm{d}}$ & ND & c. $406 \mathrm{C}>\mathrm{G}$ p.Q136E & 33 & - \\
\hline 47 & $\mathrm{~F} / 53$ & WT & ND & ND & WT $\quad 40$ & - & \\
\hline 48 & $\mathrm{~F} / 63$ & WT & ND & ND & WT & - & \\
\hline 49 & $\mathrm{~F} / 72$ & WT & ND & ND & WT & - & \\
\hline 50 & $\mathrm{~F} / 76$ & WT & $\mathrm{ND}$ & ND & WT $\quad 7$ & & \\
\hline 51 & $\mathrm{M} / 76$ & 9.2 & $\begin{array}{l}\text { a) } \mathrm{r} .461 \mathrm{~g}>\mathrm{u}(3 / 9)^{\mathrm{d}, \mathrm{g}} \\
\text { B) - }(0 / 9)^{\mathrm{g}}\end{array}$ & $\begin{array}{l}\text { A) c. } 461 \mathrm{G}>\mathrm{T}^{\mathrm{e}} \\
\text { B) c.557_558insA } \\
\text { GCTGCTCAG } \\
\text { ATAGCGA }^{\mathrm{e}}\end{array}$ & $\begin{array}{l}\text { A) c. } 461 \mathrm{G}>\mathrm{T} 4 \\
\text { B) c. } 557 \text { 558insAGCTG } \\
\text { CTCAGATAGCGA } \\
\text { A) p.G154V } \\
\text { B) p.G187Afs*67 }\end{array}$ & - & \\
\hline 52 & $\mathrm{M} / 58$ & 23.1 & r. $.533 a>u^{d}$ & ND & c.533A>T p.H178L & 36 & - \\
\hline 53 & $\mathrm{~F} / 60$ & 98.3 & r. $711 \mathrm{~g}>\mathrm{u}^{\mathrm{c}}$ & ND & c.711G>T p.M237I & 3 & ++ \\
\hline 54 & $\mathrm{M} / 69$ & 90.2 & r. $512 \mathrm{a}>\mathrm{g}^{\mathrm{c}}$ & ND & c. $512 \mathrm{~A}>\mathrm{G}$ p.E171G-ts & 4 & - \\
\hline 55 & $\mathrm{M} / 61$ & WT & ND & $\mathrm{WT}^{\mathrm{e}}$ & WT $\quad 7$ & - & \\
\hline 56 & $\mathrm{M} / 65$ & WT & ND & $\mathrm{WT}^{\mathrm{e}}$ & WT & - & \\
\hline 57 & $\mathrm{~F} / 71$ & 95.7 & $\mathrm{r} .470 \mathrm{u}>\mathrm{g}^{\mathrm{c}, \mathrm{d}}$ & c. $470 \mathrm{~T}>\mathrm{G}^{\mathrm{f}}$ & c. $.470 \mathrm{~T}>\mathrm{G}$ p.V157G & 7 & +++ \\
\hline 58 & $\mathrm{~F} / 65$ & 65.9 & $\mathrm{r} .844 \mathrm{c}>\mathrm{u}^{\mathrm{c}}$ & c. $844 \mathrm{C}>\mathrm{T}^{\mathrm{e}}$ & c. $844 \mathrm{C}>\mathrm{T}$ p.R282W & 10 & +++ \\
\hline 59 & $\mathrm{~F} / 70$ & 86.6 & r. $839 \mathrm{~g}>\mathrm{c}^{\mathrm{c}}$ & c. $839 \mathrm{G}>\mathrm{C}^{\mathrm{e}}$ & c.839G >C p.R280T & 6 & +++ \\
\hline 60 & $\mathrm{M} / 76$ & WT & ND & $\mathrm{WT}^{\mathrm{e}}$ & WT $\quad 30$ & - & \\
\hline 61 & $\mathrm{~F} / 86$ & WT & ND & ND & WT & - & \\
\hline 62 & $\mathrm{~F} / 75$ & WT & ND & ND & WT $\quad 63$ & - & \\
\hline 63 & $\mathrm{~F} / 50$ & 14.7 & r.961a $>u^{d}$ & c. $961 \mathrm{~A}>\mathrm{T}^{\mathrm{f}}$ & c. $961 \mathrm{~A}>\mathrm{T}$ p.K321* & 8 & - \\
\hline 64 & $\mathrm{M} / 63$ & WT & ND & ND & WT 6 & - & \\
\hline 65 & F74 & WT & WT & WT & WT $\quad 59$ & - & \\
\hline 66 & $\mathrm{M} / 78$ & 51.6 & r.176_375del $\mathrm{d}^{\mathrm{c}, \mathrm{d}}$ & c. $375+2 \mathrm{~T}>\mathrm{A}^{\mathrm{f}}$ & c. $375+2 \mathrm{~T}>$ A p.G59Vfs $* 23$ & 62 & - \\
\hline 67 & $\mathrm{M} / 62$ & WT & ND & ND & WT $\quad 65$ & - & \\
\hline 68 & $\mathrm{~F} / 67$ & WT & ND & ND & WT $\quad 7$ & - & \\
\hline 69 & $\mathrm{~F} / 41$ & 99.9 & r. $722 c>u^{c}$ & c. $722 \mathrm{C}>\mathrm{T}^{\mathrm{e}}$ & c. $722 \mathrm{C}>\mathrm{T}$ p.S241F & 6 & +++ \\
\hline 71 & $\mathrm{M} / 62$ & 62.1 & $\mathrm{r} .824 \mathrm{~g}>\mathrm{u}^{\mathrm{c}}$ & c. $824 \mathrm{G}>\mathrm{T}^{\mathrm{e}}$ & c. $824 \mathrm{G}>\mathrm{T}$ p.C275F & 4 & +++ \\
\hline 73 & $\mathrm{~F} / 64$ & 83.4 & $\mathrm{r} .644 \mathrm{~g}>\mathrm{c}^{\mathrm{c}, \mathrm{d}}$ & c. $644 \mathrm{G}>\mathrm{C}^{\mathrm{e}}$ & c. $644 \mathrm{G}>\mathrm{C}$ p.S215T-ts & 10 & ++ \\
\hline
\end{tabular}


Table II. Continued.

\begin{tabular}{|c|c|c|c|c|c|c|c|}
\hline \multirow[b]{2}{*}{ Case A } & \multirow[b]{2}{*}{ Gender/age } & \multirow[b]{2}{*}{ FASAY $^{\mathrm{a}}$} & \multicolumn{2}{|c|}{ DNA sequencing } & \multirow[b]{2}{*}{ TP53 status } & \multirow[b]{2}{*}{$\mathrm{FISH}^{\mathrm{b}}$} & \multirow[b]{2}{*}{ IB } \\
\hline & & & cDNA & gDNA & & & \\
\hline 74 & $\mathrm{M} / 60$ & 86.5 & $\mathrm{r} .488 \mathrm{a}>\mathrm{g}^{\mathrm{c}}$ & c. $.488 \mathrm{~A}>\mathrm{G}^{\mathrm{e}}$ & c. $488 \mathrm{~A}>$ G p.Y163C & 14 & +++ \\
\hline 75 & $\mathrm{M} / 76$ & WT & ND & ND & WT $\quad 20$ & - & \\
\hline 76 & $\mathrm{M} / 66$ & WT & ND & ND & WT & - & \\
\hline 77 & $\mathrm{~F} / 74$ & 85.4 & $\mathrm{r} .848 \mathrm{~g}>\mathrm{c}^{\mathrm{c}}$ & ND & c.848G >C p.R283P & 10 & +++ \\
\hline 78 & $\mathrm{~F} / 65$ & WT & ND & ND & WT $\quad 84$ & - & \\
\hline 79 & $\mathrm{M} / 69$ & 46.7 & $\mathrm{r} .814 \mathrm{~g}>\mathrm{u}^{\mathrm{d}}$ & c. $814 \mathrm{G}>\mathrm{T}^{\mathrm{e}, \mathrm{f}}$ & c.814G $>$ T p.V272L-ts & 10 & - \\
\hline 80 & $\mathrm{M} / 60$ & WT & ND & ND & WT $\quad 8$ & - & \\
\hline 81 & $\mathrm{M} / 69$ & 91.2 & r. $659 \mathrm{a}>\mathrm{g}^{\mathrm{c}}$ & c. $659 A>G^{f}$ & c.659A>G p.Y220C & 73 & +++ \\
\hline 82 & $\mathrm{M} / 71$ & 72.9 & r.997_1003del ${ }^{\mathrm{c}, \mathrm{d}}$ & c.997_1003del ${ }^{\mathrm{f}}$ & c.997_1003del p.R333fs*10 & 7 & ++ \\
\hline 83 & $\mathrm{~F} / 59$ & 16.8 & WT & WT & WT $\quad 52$ & - & \\
\hline 84 & $\mathrm{M} / 69$ & 64.5 & $\begin{array}{l}\text { r.176_375del } \\
(1 / 8)^{\mathrm{c}, \mathrm{d}, \mathrm{g}} \\
\text { r.[375+1g>u; } \\
375 \_376 i n s 375 \\
\left.+1 \_375+109\right] \\
(2 / 8)^{\mathrm{c}, \mathrm{d}, \mathrm{g}}, \\
\text { r.[375+1g>u; } \\
375 \_376 \text { ins } 375 \\
\left.+1 \_376-1\right] \\
(5 / 8)^{\mathrm{c}, \mathrm{d}, \mathrm{g}}\end{array}$ & c. $375+1 G>T^{f}$ & $\begin{array}{l}\text { c. } 375+1 G>\text { T } 3 \\
\text { p.G59Vfs } 23(3 / 15)^{\text {d }} \\
\text { p.Y126Vfs*59(3/15) } \\
\text { p.Y126Vfs } * 49(3 / 15)^{\text {d }}\end{array}$ & - & \\
\hline 85 & $\mathrm{~F} / 55$ & 93.5 & $\mathrm{r} .473 \mathrm{~g}>\mathrm{u}^{\mathrm{c}}$ & c. $473 \mathrm{G}>\mathrm{T}^{\mathrm{e}}$ & c.473G > T p.R158L & 36 & +++ \\
\hline 86 & $\mathrm{M} / 63$ & 98.2 & $\mathrm{r} .843 \mathrm{c}>\mathrm{g}^{\mathrm{c}}$ & c. $843 \mathrm{C}>\mathrm{G}^{\mathrm{e}}$ & c.843C>G p.D281E & 10 & +++ \\
\hline
\end{tabular}

M, male; F, female; IB, immunoblotting; ND, not done; WT, wild-type; fraction of positive colonies; ${ }^{\circ} \%$ of red colonies; ${ }^{\text {b }} \%$ of positive cells; ${ }^{\mathrm{c}} \mathrm{CDNA}$ prepared from tumor tissue; ${ }^{\mathrm{d}} \mathrm{CDNA}$ isolated from yeast colonies; ${ }_{\mathrm{e}}^{\mathrm{g} D N A}$ isolated from FFPE; ${ }_{\mathrm{f} D N A}$ isolated from frozen tumor tissue; gfraction of positive colonies.

Collection of TP53 mutations. Altogether we detected 64 TP53 mutations in 63 lung cancer cases (Tables I and II). Thirty-two mutations were detected in 32 cases of squamous cell carcinoma. Frequency of lung SQCC cases with TP53 mutation (32/42) thus reached 76.2\%. Among 56 lung AC cases, 31 were TP53 mutation positive representing 55.4\%. One AC case contained two independent TP53 mutations. Majority of the mutations (49/64) were confirmed by both cDNA and gDNA analyses. Thirteen mutations were detected by cDNA-based analyses and were not further analyzed by gDNA sequencing. Two mutations (A23-B and S41) were found by gDNA analysis but were not recognized by cDNA based techniques. The majority of detected TP53 mutations were missense. They represented $59.4 \%(19 / 32)$ of mutations detected in lung SQCC and $71.9 \%(23 / 32)$ of mutations detected in AC. Three mutations (p.E171G, p.S215T and p.V272L), all among AC cases, were recognized as temperature sensitive according to the typical phenotype of positive yeast colonies. We further detected 7 mutations affecting splicing (4 in SQCC, 3 in AC), 5 nonsense mutations ( 3 in SQCC, 2 in AC), and 10 deletions/insertions (6 in SQCC, 4 in AC), all of them causing the reading frame shift and formation of premature termination codon.

In collection of the 64 TP53 mutations, some were repeatedly detected. The p.G154V mutation appeared in four cases
(1 AC, 3 SQCCs), p.R283P in three cases (1 AC, 2 SQCC), and mutations p.Y163C, p.R158L, p.V274F and p.R280T were detected in two cases. Notably, less frequent mutation c.314G $>\mathrm{T}$ was also detected twice (A7 and S15), once in SQCC and once in $\mathrm{AC}$ case. This mutation potentially creates a new donor splice site (GT). Altogether, we sequenced the cDNA isolated from 28 yeast colonies (13 from case A7 and 15 from S15). Only single colony (case A7) revealed the presence of the transcript carrying missense mutation $\mathrm{r} .314 \mathrm{~g}>\mathrm{u}$ (p.G105V), the rest of 27 colonies contained the alternatively spliced variant r.313_375del (p.G105_T125).

Furthermore, two splice site mutations with the same effect on splicing of intron 4 were detected: mutation c.375+2T $>\mathrm{A}$ in AC sample A38, and mutation c.375+1G $>$ T in AC sample A54. Sequencing performed on cDNA from the colonies obtained by FASAY proved that these mutations indeed affected splicing. We detected even three different aberrant transcripts: i) partial deletion of exon 4, ii) insertion of 109 nucleotides of intron 4, and iii) insertion of whole intron 4 . Moreover, intronic variant c.375+5G $>$ A detected in SQCC sample S35 was shown to have the same effect on splicing although the nucleotide change did not directly affect in the consensus splice site.

Analysis of the p53 protein accumulation. We examined the p53 protein accumulation in tumor tissues of all enrolled cases 
by immunoblotting using the p53-specific DO-1 monoclonal antibody (IB). Among 42 cases of SQCC, 18 (42.9\%) were strongly positive $(+++)$ or positive $(++)$ providing intermediate level of p53. Twenty-four samples (57.1\%) exhibited low (+) or none level of the p53 protein (-). Concordance between presence of the TP53 mutation and the $\mathrm{p} 53$ protein accumulation occurred in 29 cases $(69.0 \%)$. All 18 samples positive for the p53 protein $(+++$ or ++$)$ exhibited clonal TP53 mutation. From 24 negative samples (+ or -), 13 cases featured truncating TP53 mutation, including 9 frameshifts and 1 splicing mutation. Among 56 AC cases, 19 (33.9\%) were positive (+++ or $++)$. All of them exhibited clonal TP53 mutation. Thirtyseven samples $(66.1 \%)$ were negative (+ or -). Among them, 12 samples featured truncating TP53 mutation including 5 frameshift and 2 splicing mutations. Concordance between TP53 mutational status and p53 protein accumulation occurred in $44 \mathrm{AC}$ cases $(78.6 \%)$.

FISH. We performed FISH analysis of the TP53 alleles using the locus-specific and centromeric probes. We analyzed 61 cases. Three cases (1 SQCC and 2 AC) were excluded from analysis due to suboptimal tissue fixation. Among SQCC cases, $18(43.9 \%)$ were negative as the portion of positive nuclei did not reach the $15 \%$ cut-off level. Fifteen of them featured presence and three absence of the TP53 mutation. In 23 cases $(56.1 \%)$, the TP53-specific locus was clearly lost. The portion of positive nuclei ranged from 26 to $81 \%$. The FISHpositivity was detected in 16 cases having the TP53 mutation in the other allele and in 7 cases with standard TP53 gene. Among adenocarcinomas, 20 cases $(37.0 \%)$ were positive and $34(63.0 \%)$ negative. From positive cases, 9 cases contained the TP53 mutation and 11 cases did not. Among negative cases, 21 featured the TP53 mutation while 13 standard TP53 gene. In total, cDNA/gDNA analyses did not detect any TP53 mutation in 18 cases positive in FISH: 7 SQCCs and 11 ACs.

Correlation between the p53 status and the disease outcome. In this part of the analysis, only patients with complete clinical records were included. For cohort of 36 patients with SQCC the median follow-up was 22.8 months (ranging from 0.3 to 77.2 months), the median follow-up to progression (PFS) was 21.8 months (0.3-76.9 months). For cohort of $46 \mathrm{AC}$ patients the median follow-up was 24.4 months (2.5-103.5 months), the median follow-up to progression was 21.4 months (2.0-103.5 months). We examined relation between the disease outcome (progression-free survival and overall survival) and the TP53 status: namely presence of the TP53 mutation, type of the TP53 mutation (missense vs. non-missense), loss of the TP53 locus, the p53 protein accumulation or any p53 aberration. We did not find any statistically significant difference in survival rate (both OS and PFS) in relation to any examined factor but some trends for their relationship were demonstrated. The most pronounced association was found between the presence of any p53 aberration in tumor tissue and decreased PFS $(\mathrm{p}=0.074)$ and $\mathrm{OS}(\mathrm{p}=0.121)$ of patients with lung AC.

\section{Discussion}

In the present study, we performed detailed analyses of the TP53 status in 98 cases of lung carcinoma. Our cohort comprised of 42 cases of squamous cell carcinoma and 56 cases of lung adenocarcinoma (AC). We analyzed cases which were sequentially collected in tissue bank of the University Hospital Brno from March 2006 to January 2014. Parameters of our cohort (e.g. age, male/female, SQCC/AC and non-smokers/smokers ratios) are similar to corresponding cohorts worldwide. Results of our analysis are in good agreement with previous findings. Proportion of the TP53 mutation-positive cases is very high among our lung AC and particularly among SQCC cases reaching 55.4 and $76.2 \%$, respectively. Frequency of cases with any somatic TP53 aberrations, mutation and/or deletion, is even higher reaching $75 \%$ for $\mathrm{AC}$ and $92.9 \%$ for SQCC cases. To some extent, it corresponds to the results of the comprehensive studies describing $34 \%$ of mutated cases for lung AC (15) and $90 \%$ for lung SQCC (14). The complex and detailed analysis employing several methodically diverse approaches and particularly powerful and highly sensitive FASAY are the likely reasons for more efficient detection of the TP53 aberrations in our study. FASAY has been repeatedly shown to be potent and robust tool for detection and further analyses of TP53 mutations (21). In the present study, we carefully searched for a TP53 mutation in 12 cases classified as wildtype by FASAY. Using cDNA and gDNA sequencing we found the TP53 mutation in only one of them (AC case A23). In fact, two different TP53 mutations were traced in the sample. One of them (A23-B: c.557_558insAGCTGCTCAGATAGCGA) caused frame shift and formation of premature termination codon and mRNA degradation which, in combination with its low prevalence in sample $(\sim 15 \%$ based on gDNA sequencing estimation), explains why it was missed by FASAY. The other mutation found in the same sample by gDNA sequencing (A23-A: p.G154V)-which should be detectable by FASAY-had comparably low prevalence in the sample. This one initially unrevealed and then revised positive case represents $8 \%$ from 12 comprehensively reassessed TP53 mutation-negative cases providing an estimation of false negativity of FASAY. Similarly, the nonsense mutations could also be missed in the examination. In case of the mutation p.E171* (case S41) only our examination of another suspected (and finally not confirmed) mutation resulted in its disclosure. Detection of the mutation p.K321* in AC sample A35 was also difficult. It was detected in only 3 from 18 red yeast colonies and subsequently confirmed by gDNA sequencing. The mutation p.E171* has already been reported 22 times in tumors, notably 10 times just in lung carcinomas. The mutation p.K321* is rather rare as it has been previously reported only twice and never in lung carcinoma (http://www-p53.iarc.fr; R17; 20). Nevertheless, FASAY again proved to be considerably successful even in detection of the TP53 mutations associated with nonsense-mediated mRNA decay as demonstrated by other cases, for example SQCC cases S4, S23, S38, AC cases A10, A16 and others. Altogether, we detected 16 mutations leading to formation of premature termination codon. Five mutations were nonsense and 11 mutations were deletions and/ or insertions causing reading frame shift. These mutations represent as much as $25 \%$ of all detected mutations in our whole cohort and even $31.25 \%$ (10/32) among SQCC cases (while only $18.75 \%-6 / 32$ in AC cases).

Inactivation of $\mathrm{p} 53$ usually results from a missense mutation in the core region of the TP53 gene coding for the sequence- 
specific DNA-binding domain. According to the IARC database, proportion of the missense mutations represents $73.2 \%$ on average and $72.8 \%$ in lung tumors (http://www-p53.iarc.fr; R17; 20). In our samples, missense mutations represented $71.9 \%$ of mutations detected in AC but occurred less frequently in SQCC representing only $59.4 \%$. An interesting, though missense, mutation was detected in AC sample A15: p.R248L. Its determination was unequivocal but quite surprising as the mutation represents exchange of dinucleotide (c.743_744GG>TT). Nevertheless, the mutation has already been reported four times, three of them in lung carcinoma. This mutation, similarly to p.E171* seems to occur rarely but predominantly in lung carcinoma (http:// www-p53.iarc.fr; R17; 20).

We repeatedly detected several mutations, namely p.G154V (4x), p.R283P (3x), p.Y163C, p.R158L, p.V274F, p.R280T and mutation p.[G105V, G105_T125del]. Among them, only mutations in codons 158 and 163 were found repeatedly in other cohorts $(14,22)$. On the contrary, mutations suggested as lung carcinoma hot-spots by other authors were also found by us, namely mutations in codons $155,245,248,275,282$ and also in codon 157 (AC case A29) which is considered to be associated with smoking $(11,14,23,24)$. From mutations detected repeatedly in our cohort, the mutation c.314G $>\mathrm{T}$ was the most unusual. The mutation, though not typical for the TP53 gene and occurring with low frequency, appeared twice: in SQCC case S15 and in AC case A7. Though the analyses of these cases were not performed at the same time, we were aware of risk of sample swapping. To rule out this possibility, both cases were analyzed repeatedly, both providing consistently the same results. We observed that while the case S15 was heterozygous in polymorphism p.P72R/p.P72P, the case A7 was homozygous p.P72R. This convinced us that indeed the two samples correspond to two different persons. In addition, this conclusion was unequivocally verified by the chimerism test. According the IARC TP53 database (http://www-p53. iarc.fr; R17; 20), the mutation c.314G $>$ T has predicted its effect on splicing. The FASAY, as its full name (functional analysis of separated alleles in yeast) indicates it provides the way to assess frequency of particular TP53 alleles. Indeed, using FASAY we were able to detect the alternatively spliced transcript p.G105_T125del, which was prevalent in both cases (12/13 and 15/15 colonies, respectively).

Notably, we found three different splicing mutations in intron 4 (c. $375+5 \mathrm{G}>\mathrm{A}$, c. $375+2 \mathrm{~T}>\mathrm{A}, \mathrm{c} .375+1 \mathrm{G}>\mathrm{T})$ resulting in creation of multiple aberrant transcripts in all cases. All the three splicing mutations are rather rare, but each has been already reported: once, five times and once, respectively; none of them in lung carcinoma (http://www-p53.iarc.fr; R17; 20).

We detected accumulation of the p53 protein in $57.1 \%$ of SQCC cases and $33.9 \%$ of AC cases. This result fits well with other studies $(11,25)$. Similarly, the concordance we found between the TP53 mutations and the p53 protein accumulation for SQCC cases (69.0\%) and AC cases (78.6\%) is comparable to published data (60 and 70\%) (11). Notably, the level of concordance would be even higher if the type of the TP53 mutation was taken into account. We clearly showed that several non-concordant p53 proteins, negative/TP53 mutation-positive cases, contained mutation causing formation of premature termination codon and mRNA degradation. In contrast, the concordance between TP53 mutation and loss of the TP53 specific locus $17 \mathrm{p} 13$ was considerable lower reaching 46.3\% in SQCC and 40.7\% in AC. However, it has been also previously shown that in NSCLC the TP53 mutations appear both with and without the allele loss (11). Our results confirm this observation and show that the TP53 allele loss appears both with and without TP53 mutation. It indicates the overall high loss of the p53 function in lung carcinomas confirming its importance in process of the lung carcinoma development and progression.

Several studies, including a large meta-analysis showed that abnormal p53 expression was associated with decreased survival across all stages in NSCLC in both adenocarcinoma and squamous cell carcinoma $(11,26,27)$. However, results of our study did not support this conclusion. Although we examined relation between the disease outcome and several p53 parameters as presence and type of the TP53 mutation, loss of the TP53 locus, the p53 protein accumulation or p53 aberration, we did not find any statistically significant difference. The insufficient size of the cohort of patients can provide one possible explanation for this discrepancy. Due to high proportion of cases positive for a 553 aberration, the subgroup without a p53 hit was not sufficient. It may also explain why the only association coming close to statistical significance concerned the $\mathrm{AC}$ cases among which the proportion of p53 aberrations was lower. In addition, similar results demonstrating p53 as significant prognostic marker in AC but not squamous cell carcinoma has been reported earlier $(10,27)$.

In conclusion, although our results failed to boost the role of the p53 inactivation as a negative prognostic marker in lung carcinoma, they clearly support its critical role in lung carcinoma development. Last but not least, we demonstrated the usefulness and convenience of FASAY as a fast, simple and reliable instrument for detection of TP53 mutations in human tissues.

\section{Acknowledgements}

The present study was supported by grant NT/13784-4/2012 of the Internal Grant Agency of the Ministry of Health of the Czech Republic, and by MH CZ-DRO (FNBr, 65269705). We thank Dr Ondrej Horky from UH, Brno for performing the chimerism test.

\section{References}

1. Siegel R, Naishadham D and Jemal A: Cancer statistics, 2013. CA Cancer J Clin 63: 11-30, 2013.

2. Siegel R, Ma J, Zou Z and Jemal A: Cancer statistics, 2014. CA Cancer J Clin 64: 9-29, 2014.

3. Harris SL and Levine AJ: The p53 pathway: Positive and negative feedback loops. Oncogene 24: 2899-2908, 2005.

4. Sherr CJ: Principles of tumor suppression. Cell 116: 235-246, 2004.

5. Haupt Y, Maya R, Kazaz A and Oren M: Mdm2 promotes the rapid degradation of p53. Nature 387: 296-299, 1997.

6. Honda R, Tanaka H and Yasuda H: Oncoprotein MDM2 is a ubiquitin ligase E3 for tumor suppressor p53. FEBS Lett 420: 25-27, 1997.

7. Wu X, Bayle JH, Olson D and Levine AJ: The p53-mdm-2 autoregulatory feedback loop. Genes Dev 7: 1126-1132, 1993.

8. Lin MW, Wu CT, Shih JY, Chang YL and Yang PC: Clinicopathologic characteristics and prognostic significance of $E G F R$ and $p 53$ mutations in surgically resected lung adenocarcinomas $\leq 2 \mathrm{~cm}$ in maximal dimension. J Surg Oncol 110: 99-106, 2014.

9. Campling BG and El-Deiry WS: Clinical implication of p53 mutation in lung cancer. Mol Biotechnol 24: 141-156, 2003. 
10. Huang CL, Yokomise $\mathrm{H}$ and Miyatake A: Clinical significance of the $\mathrm{p} 53$ pathway and associated gene therapy in non-small cell lung cancers. Future Oncol 3: 83-93, 2007.

11. Mogi A and Kuwano H: TP53 mutations in nonsmall cell lung cancer. J Biomed Biotechnol 2011: 583929, 2011

12. Kishimoto Y, Murakami Y, Shiraishi M, Hayashi K and Sekiya T: Aberrations of the $p 53$ tumor suppressor gene in human non-small cell carcinomas of the lung. Cancer Res 52: 4799-4804, 1992.

13. Tammemagi MC, McLaughlin JR and Bull SB: Meta-analyses of $p 53$ tumor suppressor gene alterations and clinicopathological features in resected lung cancers. Cancer Epidemiol Biomarkers Prev 8: 625-634, 1999.

14. Ding L, Getz G, Wheeler DA, Mardis ER, McLellan MD, Cibulskis K, Sougnez C, Greulich H, Muzny DM, Morgan MB, et al: Somatic mutations affect key pathways in lung adenocarcinoma. Nature 455: 1069-1075, 2008.

15. Cancer Genome Atlas Research Network: Comprehensive genomic characterization of squamous cell lung cancers. Nature 489: 519-525, 2012.

16. Flaman JM, Frebourg T, Moreau V, Charbonnier F, Martin C, Chappuis P, Sappino AP, Limacher IM, Bron L and Benhattar J: A simple p53 functional assay for screening cell lines, blood, and tumors. Proc Natl Acad Sci USA 92: 3963-3967, 1995.

17. Smardová J, Nemajerová A, Trbusek M, Vagunda V and Kovarík J: Rare somatic p53 mutation identified in breast cancer: A case report. Tumour Biol 22: 59-66, 2001.

18. Ishioka C, Frebourg T, Yan YX, Vidal M, Friend SH, Schmidt S and Iggo R: Screening patients for heterozygous p53 mutations using a functional assay in yeast. Nat Genet 5: 124-129, 1993.
19. Waridel F, Estreicher A, Bron L, Flaman JM, Fontolliet C, Monnier P, Frebourg T and Iggo R: Field cancerisation and polyclonal p53 mutation in the upper aero-digestive tract. Oncogene 14: 163-169, 1997.

20. Petitjean A, Mathe E, Kato S, Ishioka C, Tavtigian SV, Hainaut $P$ and Olivier M: Impact of mutant p53 functional properties on TP53 mutation patterns and tumor phenotype: Lessons from recent developments in the IARC TP53 database. Hum Mutat 28: 622-629, 2007.

21. Smardová J, Smarda J and Koptíková J: Functional analysis of p53 tumor suppressor in yeast. Differentiation 73: 261-277, 2005.

22. Toyooka S, Tsuda T and Gazdar AF: The TP53 gene, tobacco exposure, and lung cancer. Hum Mutat 21: 229-239, 2003.

23. Rodin SN and Rodin AS: Origins and selection of p53 mutations in lung carcinogenesis. Semin Cancer Biol 15: 103-112, 2005

24. Gibbons DL, Byers LA and Kurie JM: Smoking, p53 mutation, and lung cancer. Mol Cancer Res 12: 3-13, 2014.

25. Xu Y, Wang L, Zheng X, Liu G, Wang Y, Lai X and Li J: Positive expression of p53, c-erbB2 and MRP proteins is correlated with survival rates of NSCLC patients. Mol Clin Oncol 1: 487-492, 2013.

26. Steels E, Paesmans M, Berghmans T, Branle F, Lemaitre F, Mascaux C, Meert AP, Vallot F, Lafitte JJ and Sculier JP: Role of p53 as a prognostic factor for survival in lung cancer: A systematic review of the literature with a meta-analysis. Eur Respir J 18: 705-719, 2001.

27. Nikliński J, Niklińska W, Laudanski J, Chyczewska E and Chyczewski L: Prognostic molecular markers in non-small cell lung cancer. Lung Cancer 34 (Suppl 2): S53-S58, 2001. 